\title{
EDITORIAL
}

\section{Have biomarkers failed in acute kidney injury? No}

\author{
Blaithin A. McMahon ${ }^{1}$ and Jay L. Koyner ${ }^{2 *}$
}

(c) 2017 Springer-Verlag Berlin Heidelberg and ESICM

Over the past decade, large prospective cohort studies have repeatedly shown that novel biomarkers are superior in performance to serum creatinine ( $\mathrm{SCr}$ ) in the early diagnosis and prognosis of acute kidney injury (AKI) with this data being summarized in recent reviews $[1,2]$. Several investigations have linked these first-generation serum and urinary biomarkers of AKI [e.g., neutrophil gelatinase-associated lipocalin (NGAL) or tissue inhibitor of metalloproteinases (TIMP)-2 and insulin-like growth factor binding protein 7 (IGFBP7)] with adverse patient outcomes even in the absence of a change in $\mathrm{SCr}$ concentrations (biomarker-"positive"/creatinine-"negative" status) $[3,4]$. We will delineate the barriers to biomarker development and validation in AKI while highlighting methods to overcome these barriers in an attempt to improve the management of patients with AKI.

While numerous novel biomarkers have been assessed in diverse populations in various AKI clinical scenarios, their implementation into current clinical practices has been hindered primarily as a result of the continued comparison to the imperfect gold standard SCr [5] but also the unclear impact of chronic kidney disease (CKD) on biomarker performance [6] and testing of a single biomarker in a heterogeneous pool of AKI (cardiac surgery or ICU-associated AKI) [7], resulting in suboptimal areas under the receiver-operating characteristic curves (AUROC). Table 1 highlights some potential solutions to these barriers of AKI biomarker development.

Validation of biomarker performance against consensus definitions of SCr defined AKI has been particularly

\footnotetext{
*Correspondence: jkoyner@medicine.bsd.uchicago.edu

${ }^{2}$ Section of Nephrology, Department of Medicine, University of Chicago, 5841 South Maryland Ave, Suite S-506, MC 5100, Chicago, IL 60637, USA Full author information is available at the end of the article
}

For contrasting viewpoints, please go to doi:10.1007/s00134-017-4759-3 and doi:10.1007/s00134-017-4763-7. problematic. Even though an increase in biomarker levels can predict an impending increase in SCr, only a change in $\mathrm{SCr}$ can diagnose AKI. This over-reliance on $\mathrm{SCr}$ has led some investigators to avoid SCr-based definitions entirely, choosing to link elevations in functional and structural biomarkers to outcomes such as the need for renal replacement therapy (RRT) and death [8]. In fact, recently proposed consensus definitions classify AKI through changes in biomarkers in the absence of changes in $\mathrm{SCr}$ [9]. While not all biomarkers have shown this phenomena, it is important to acknowledge that many of these first-generation tests are able to detect ongoing tubular injury in the setting of renal reserve before there is a change in functional/filtration capacity ( $\mathrm{SCr}$ or cystatin $\mathrm{C}$ ) and allow a window of time when interventions might be able to prevent further injury [10].

Combining biomarkers with other risk stratification techniques is yet another method in which biomarkers can help improve outcomes in AKI in high-risk patients. Adding biomarkers to AKI clinical risk models (e.g., renal angina index or cardiac surgery-associated AKI risk model) has been shown to further enhance biomarker utility [11]. Another risk stratification method that has excellent discriminatory capacity for predicting severe AKI in patients with early AKI is the furosemide stress test (FST). Assessment of urine output following an FST challenge (protocoled dose of a diuretic in the setting of early AKI) has demonstrated the ability to improve patient risk stratification for adverse outcomes (AKI progression, need for RRT, and inpatient mortality) [12]. In fact pairing functional and structural injury markers (as suggested by a recent Acute Dialysis Quality Initiative consensus paper) such as NGAL and TIMP2"IGFBP7 with the FST improves their accuracy in detecting these impending adverse events [13]. Others have shown that combining the risk stratification of the renal angina index with biomarkers enhances the detection of those

\section{望


Table 1 Methods to improve biomarker development

Need for inclusion of biomarkers in internationally accepted consensus of AKI definition
Combine use with risk stratification methods such as the renal angina index and furosemide stress test to enhance sensitivity and specificity
Link biomarkers to adverse clinical outcomes and avoid SCr use
Need for biomarker manufacturer to establish separate reference range around AKI and CKD when appropriate
Decreased reliance on SCr in the diagnosis of AKI which is influenced by muscle wasting and volume overload
Need to advance biostatistical methods around biomarker combinations

$A K I$ acute kidney injury, SCr serum creatinine, $C K D$ chronic kidney disease

at highest risk for morbidity and mortality [11]. As our understanding of these first-generation risk stratification methods improves they will expand our knowledge of appropriate biomarker use and improve the care and outcomes of those with AKI.

Biomarkers have also been shown to be informative for the inclusion of patients with AKI in intervention trials $[10,14,15]$. The PrevAKI trial is the most recent example of trials utilizing biomarkers to trigger patient randomization and we anticipate growth of this technique given these successes [10]. In the PrevAKI trial, patients with [TIMP-2] $\times$ [IGFBP7] $\left(\right.$ Nephrocheck $\left.^{\circledR}\right)$ levels greater than $0.3,4 \mathrm{~h}$ after cardiopulmonary bypass were randomized to receive a "KDIGO-based care bundle" consisting of optimization of volume status, and hemodynamics, avoidance of nephrotoxins, and prevention of hyperglycemia. Rates of AKI were significantly reduced by the bundle compared with standard care $(55.1 \%$ vs $71.1 \%$, $95 \%$ CI $5.5-27.9 \%, p=0.004$ ) [10]. As more trials incorporate biomarkers into their entry criteria and protocols we will further perfect and understand the strengths and weaknesses of these first-generation AKI biomarkers.

Are the currently available biomarkers perfect? No they are not. Their development is hindered by comparison to $\mathrm{SCr}$ and heterogeneity in AKI sources. As an example not all cardiac surgery-associated AKI is due to ischemic injury (as originally thought); some may be due to athero-embolic disease, or sepsis, or interstitial nephritis, but it all gets reported as a change in SCr. Thus each unique biomarker may not be ideally suited for the same "one-size-fits-all" model we have created with SCr. It took decades to overcome the barriers and refine the assays before the high sensitivity troponin immunoassays could be optimized for use. The association of first-generation biomarkers with clinically meaningful endpoints such as early AKI, progression of AKI, need for RRT, and long-term mortality is clear $[1,2]$. As above, a recent consensus statement included biomarkers in the definitions of AKI and given the imperfections of $\mathrm{SCr}$ and difficulty in assessing renal reserve there is increasing acceptance of the importance of the biomarker-positive creatinine-negative cohorts [9]. We must continue to use these tools and embrace them despite their limitations or lack of an AUROC $>0.90$. Just as $\mathrm{SCr}$ and urine output are imperfect biomarkers of $\mathrm{AKI}$, it is not reasonable to expect NGAL or TIMP2"IGFBP7 or any other biomarker to predict all outcomes (early AKI, severe AKI/need for RRT, mortality) in every clinical setting (emergency room, ICU, cardiac surgery). As nephrologists and intensivists continue to care for patients with AKI, they need to be aware of the clinical value of these first-generation biomarkers and when appropriate they should work to incorporate them into clinical care (e.g., use of "KDIGO bundle"). While this is occurring we need continued research in the field of critical care nephrology and we must work to discover and refine the next generation of AKI biomarkers.

\section{Author details \\ ${ }^{1}$ Division of Nephrology, Johns Hopkins University School of Medicine, 1830 East Monument St, Suite 416, Baltimore, MD 21287, USA. ${ }^{2}$ Section of Nephrol- ogy, Department of Medicine, University of Chicago, 5841 South Maryland Ave, Suite S-506, MC 5100, Chicago, IL 60637, USA.}

\section{Compliance with ethical standards}

\section{Conflicts of interest}

BAM has no disclosures. JLK reports research support from Astute Medical, Satellite Health Care and NxStage as well as receiving consulting fees from Astute Medical and Sphingotec

Received: 2 February 2017 Accepted: 1 April 2017

Published online: 24 April 2017

\section{References}

1. Malhotra R, Siew ED (2017) Biomarkers for the early detection and prognosis of acute kidney injury. Clin J Am Soc Nephrol 12:149-173

2. McMahon BA, Koyner JL (2016) Risk stratification for acute kidney injury: are biomarkers enough? Adv Chronic Kidney Dis 23:167-178

3. Nickolas TL, Schmidt-Ott KM, Canetta P, Forster C, Singer E, Sise M, Elger A, Maarouf O, Sola-Del Valle DA, O'Rourke M, Sherman E, Lee P, Geara A, Imus P, Guddati A, Polland A, Rahman W, Elitok S, Malik N, Giglio J, ElSayegh S, Devarajan P, Hebbar S, Saggi SJ, Hahn B, Kettritz R, Luft FC, Barasch J (2012) Diagnostic and prognostic stratification in the emergency department using urinary biomarkers of nephron damage: a multicenter prospective cohort study. J Am Coll Cardiol 59:246-255

4. Ronco C, Kellum JA, Haase M (2012) Subclinical AKI is still AKI. Crit Care 16:313

5. Waikar SS, Betensky RA, Emerson SC, Bonventre JV (2012) Imperfect gold standards for kidney injury biomarker evaluation. J Am Soc Nephrol 23:13-21 
6. Koyner JL, Coca SG, Thiessen-Philbrook H, Patel UD, Shlipak MG, Garg AX, Parikh CR (2015) Urine biomarkers and perioperative acute kidney injury: the impact of preoperative estimated GFR. Am J Kidney Dis 66:1006-1014

7. Endre ZH, Pickering JW, Walker RJ, Devarajan P, Edelstein CL, Bonventre JV, Frampton CM, Bennett MR, Ma Q, Sabbisetti VS, Vaidya VS, Walcher AM, Shaw GM, Henderson SJ, Nejat M, Schollum JB, George PM (2011) Improved performance of urinary biomarkers of acute kidney injury in the critically ill by stratification for injury duration and baseline renal function. Kidney Int 79:1119-1130

8. Pickering JW, Endre ZH (2013) Linking injury to outcome in acute kidney injury: a matter of sensitivity. PLoS One 8:e62691

9. Chawla LS, Bellomo R, Bihorac A, Goldstein SL, Siew ED, Bagshaw SM, Bittleman D, Cruz D, Endre Z, Fitzgerald RL, Forni L, Kane-Gill SL, Hoste E, Koyner J, Liu KD, Macedo E, Mehta R, Murray P, Nadim M, Ostermann M, Palevsky PM, Pannu N, Rosner M, Wald R, Zarbock A, Ronco C, Kellum JA (2017) Acute kidney disease and renal recovery: consensus report of the Acute Disease Quality Initiative (ADQI) 16 Workgroup. Nat Rev Nephrol 13:241-257

10. Meersch M, Schmidt C, Hoffmeier A, Van Aken H, Wempe C, Gerss J, Zarbock A (2017) Prevention of cardiac surgery-associated AKI by implementing the KDIGO guidelines in high risk patients identified by biomarkers: the PrevAKI randomized controlled trial. Intensive Care Med. doi:10.1007/s00134-016-4670-3
11. Basu RK, Wang Y, Wong HR, Chawla LS, Wheeler DS, Goldstein SL (2014) Incorporation of biomarkers with the renal angina index for prediction of severe AKI in critically ill children. Clin J Am Soc Nephrol 9:654-662

12. Chawla LS, Davison DL, Brasha-Mitchell E, Koyner JL, Arthur JM, Shaw AD, Tumlin JA, Trevino SA, Kimmel PL, Seneff MG (2013) Development and standardization of a furosemide stress test to predict the severity of acute kidney injury. Crit Care 17:R207

13. Koyner JL, Davison DL, Brasha-Mitchell E, Chalikonda DM, Arthur JM, Shaw AD, Tumlin JA, Trevino SA, Bennett MR, Kimmel PL, Seneff MG, Chawla LS (2015) Furosemide stress test and biomarkers for the prediction of AKI severity. J Am Soc Nephrol 26:2023-2031

14. Zarbock A, Kellum JA, Schmidt C, Van Aken H, Wempe C, Pavenstadt H, Boanta A, Gerss J, Meersch M (2016) Effect of early vs delayed initiation of renal replacement therapy on mortality in critically III patients with acute kidney injury: the ELAIN randomized clinical trial. JAMA 315:2190-2199

15. Smith OM, Wald R, Adhikari NK, Pope K, Weir MA, Bagshaw SM (2013) Standard versus accelerated initiation of renal replacement therapy in acute kidney injury (STARRT-AKI): study protocol for a randomized controlled trial. Trials 14:320 